\title{
MONITORING CAPABILITIES OF A MOBILE MAPPING SYSTEM BASED ON NAVIGATION QUALITIES
}

\author{
H. Jing ${ }^{\mathrm{a}, \mathrm{b} *}$, N. Slatcher ${ }^{\mathrm{a}}$, X. Meng ${ }^{\mathrm{b}}$, G. Hunter ${ }^{\mathrm{a}}$
}

a 3D Laser Mapping Ltd, Bingham, Nottinghamshire, UK - (haojing, Neil.Slatcher, Graham.Hunter)@ 3dlasermapping.com

${ }^{\mathrm{b}}$ Nottingham Geospatial Institute, University of Nottingham, Nottingham, UK - (Julia.Jing, Xiaolin.Meng)@ nottingham.ac.uk

\author{
Commission I, ICWG I/Va
}

KEY WORDS: Mobile mapping, Navigation, Change detection, Monitoring

\begin{abstract}
:
Mobile mapping systems are becoming increasingly popular as they can build 3D models of the environment rapidly by using a laser scanner that is integrated with a navigation system. 3D mobile mapping has been widely used for applications such as 3D city modelling and mapping of the scanned environments. However, accurate mapping relies on not only the scanner's performance but also on the quality of the navigation results (accuracy and robustness). This paper discusses the potentials of using 3D mobile mapping systems for landscape change detection, that is traditionally carried out by terrestrial laser scanners that can be accurately geo-referenced at a static location to produce highly accurate dense point clouds. Yet compared to conventional surveying using terrestrial laser scanners, several advantages of mobile mapping systems can be identified. A large area can be monitored in a relatively short period, which enables high repeat frequency monitoring without having to set-up dedicated stations. However, current mobile mapping applications are limited by the quality of navigation results, especially in different environments. The change detection ability of mobile mapping systems is therefore significantly affected by the quality of the navigation results. This paper presents some data collected for the purpose of monitoring from a mobile platform. The datasets are analysed to address current potentials and difficulties. The change detection results are also presented based on the collected dataset. Results indicate the potentials of change detection using a mobile mapping system and suggestions to enhance quality and robustness.
\end{abstract}

\section{INTRODUCTION}

Change detection methods have evolved greatly over the years, from conventional remote sensing techniques to using laser scanners, also known as light detection and ranging (LiDAR). Terrestrial LiDAR is able to generate high resolution point clouds of the scanned environment, thus can be used to monitor specific objects with great detail and accuracy, such as landslides and infrastructure deformation monitoring (Park et al., 2007). With the advances in navigation systems, LiDAR applications have evolved with better flexibity by putting the laser scanner on mobile platforms, i.e. airborne LiDAR and mobile LiDAR, or mobile laser scanning system (MLS). These systems have the advantages of being able to survey larger areas over a relatively short period of time with high position accuracy and precision in three dimensions (3D). Both terrestrial and mobile systems are similar in that the positions of the point clouds measured by the scanner are both computed in a local scanner coordinate system which only indicates the relative measurement from the scanner to the target (Lindenbergh and Pietrzyk, 2015). The absolute position of the laser scanner is provided by a navigation system which is integrated with the point cloud during post-processing. Therefore, the accuracy of the point cloud depends on the positioning accuracy of the navigation system. Compared to airborne applications, MLS is able to capture more details of the environment as it is much closer to the targets, hence often used in mobile mapping applications to build 3D models (Kaartinen et al., 2012). Recent improvements in both navigation system accuracy and laser scanner performance allows the applications of MLS based monitoring and surveying, which has a higher demand for accuracy and detail (Vu et al., 2004, Bitenc et al., 2011, González-Jorge et al., 2012).
Road and railway surveying applications have grown towards using MLS rather than traditional surveying methods as MLS can reduce safety risks by taking surveyors off the road while also generating 3D road models efficiently. These model are then used for analysing clearances, tunnel profiles, road geometry and other road-side infrastructure. Some environmental applications were also presented such as fluvial morphology and erosion detection (Kukko et al., 2010). One of the greatest benefits of using MLS for monitoring is its ability to reduce human labour throughout the whole work flow while maintaining the required accuracy and reliability (Xiao et al., 2013). However, ground control points are normally required to ensure the accuracy of the point cloud and the 3D model (Kaartinen et al., 2012), which then allows accurate measurement of 3D displacements. Yet, installing control points along the monitored area means that some level of human work is still required and the locations where control points are really needed can be very remote and difficult places.

This paper presents two case studys to discuss the change detection and monitoring capabilities using airborne and mobile LiDAR respectively. While monitoring requires the ability of measuring how much the environment has changed, which requires centimetre or even millimetre data accuracy, change detection addresses the question of whether the environment has changed or not, which does not require such high accuracy. Hence the possibility of using MLS in a number of different environments. Laser point cloud geo-referencing accuracy, the biggest challenge towards effective change detection is assessed without installing control points. The effect of navigation accuracy on geo-referencing of the point cloud and the accuracy of the change detection is analysed to understand the monitoring capabilities of mobile systems. Several potential methods are discussed to address the current challenges of monitoring using a mobile platform.

${ }^{*}$ Corresponding author 


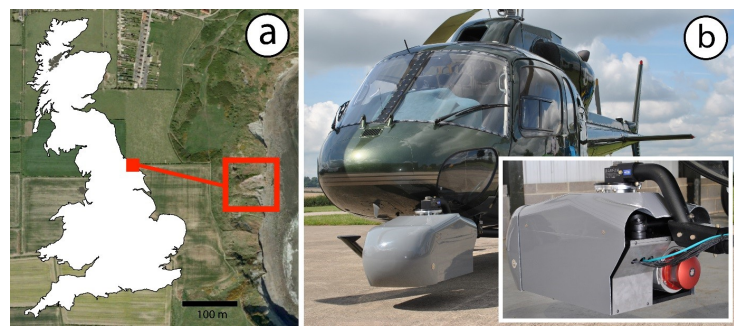

Figure 1: Airborne system and the study area

\section{DATASETS}

\subsection{Study area 1}

The first study area is a small landslide near Whitby, located on the North Yorkshire coast in the UK. The area consisted of both bare earth and vegetated terrain. The coastal cliffs in the area consist of interbedded mudstones, shales, siltstones, ironstones and sandstones that form the Staithes Sandstone and underlying Redcar Mudstone formations. This area was monitored due to significant coastal instability with regular rockfall and landslide events. Two repeat surveys were captured with a ten-month interval using 3D Laser Mapping's (3DLM) airborne mapping system that was installed on a helicopter.

The mapping system consisted of a RIEGL VQ-450 laser scanner integrated with an IGI AeroControl navigation system. The navigation system consisted of a high-end Global Navigation Satellite System (GNSS) receiver and a high accuracy Inertial Measurement Unit (IMU) which used a fibre optic gyro. The RIEGL VQ-450 laser scanner specifications are as listed in Table 1. The scanner is able to perform online-waveform processing which enables multiple target detection for each individual laser pulse. This enables more accurate modelling of the terrain, especially areas that are covered by vegetation. The system is installed on the helicopter with a $180^{\circ}$ downward and sideways looking fieldof-view. A 36.3 megapixel Nikon D-800 camera was also installed to capture optical images. The average flight height was $100 \mathrm{~m}$ which enables point spacing of around $15 \mathrm{~cm}$ and a ground sample distance (GSD) of around $1 \mathrm{~cm}$ for the camera images (Slatcher et al., 2015).

Land surface change detection and monitoring was carried out to help understand the coastal erosions and cliff movements around the area.

\subsection{Study area 2}

The second selected study area is around 10 miles along the Snake Pass, a part of the A57 road in Derbyshire, UK, between Glossop and the Ladybower Reservoir. This area has attracted the attention of the British Geological Survey (BGS), local councils and geologists due to its poor road accident record (Boon and Evans, 2008). This area is mostly undulating steep moorland used for grazing with small woods scattered along the valley bottom. It is frequently closed in winter due to snow as well as several long term closures caused by subsidence following heavy rain. As a result, road users and tourists in the area are at risk of potential dangers. More recently, the road was closed in 2012 for resurfacing and again in June 2015 for major repairs to install retaining walls. Therefore, this area was selected for frequent MLS data capture to produce a database of the road and its surrounding environment. Changes in this area are measured from the point cloud, which can later be used to provide useful information in monitoring and predicting the road conditions. The collected data is used here to assess the change detection ability of 3DLM's mobile mapping system, StreetMapper (shown in Figure 2b), based

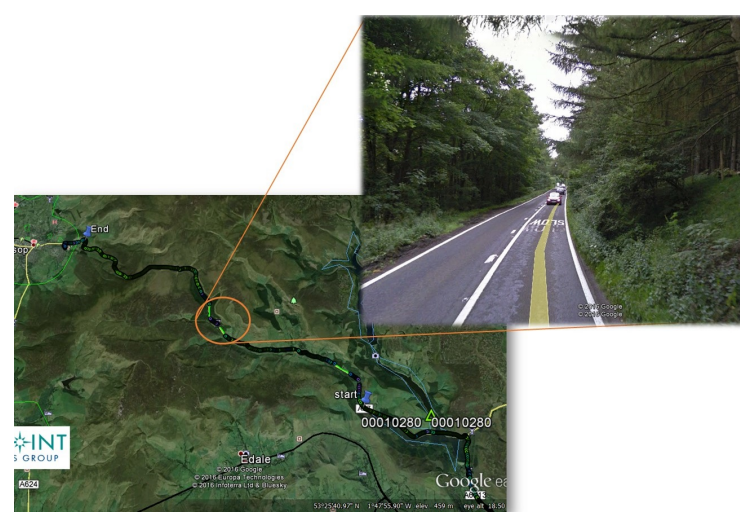

(a) Snake Pass environment

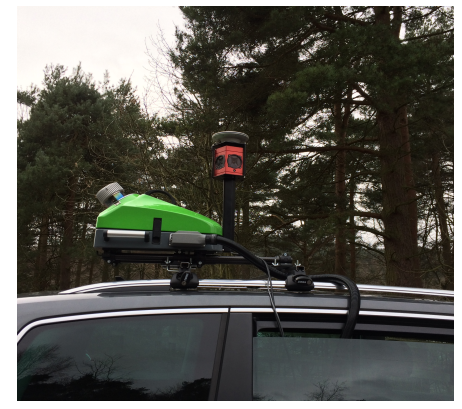

(b) Single scanner StreetMapper system

Figure 2: Environment and equipment for Case study 2

on the acquired navigation accuracy.

The StreetMapper system used in this trial consisted of a RIEGL VUX-1 laser scanner integrated with the same IGI AeroControl navigation system as mentioned in the previous data capture trial. VUX-1 is a more compact and lightweight scanner compared to VQ450 and its specifications are also listed in Table 1. The system is installed towards the back of the test vehicle roof with a $50^{\circ}$ angle to the horizontal plane, allowing the scanner to capture details of the ground and along both sides of the road.

Table 1: RIEGL laser scanner performance

\begin{tabular}{l|l|l|l}
\hline & $\begin{array}{l}\text { Max measuring } \\
\text { range }\end{array}$ & Field of view & $\begin{array}{l}\text { Measurement } \\
\text { rate }\end{array}$ \\
\hline $\begin{array}{l}\text { VQ-450 } \\
\text { VUX-1 }\end{array}$ & $\begin{array}{l}\text { up to } 800 \mathrm{~m} \\
\text { up to } 920 \mathrm{~m}\end{array}$ & $\begin{array}{l}360^{\circ} \\
330^{\circ}\end{array}$ & $\begin{array}{l}\text { up to } 550 \mathrm{kHz} \\
\text { up to 550kHz }\end{array}$ \\
\hline & $\begin{array}{l}\text { Angle measure- } \\
\text { ment resolution }\end{array}$ & Accuracy & Precision \\
\hline VQ-450 & $0.001^{\circ}$ & $8 \mathrm{~mm}$ & $5 \mathrm{~mm}$ \\
VUX-1 & $0.001^{\circ}$ & $10 \mathrm{~mm}$ & $5 \mathrm{~mm}$ \\
\hline
\end{tabular}

\subsection{The mapping systems}

Both scanners used in the case studies are 2D laser scanners, i.e. a line scan is performed and only one scan angle per laser measurement is acquired each time. The line scan can produce a nearly straight line or a 1D curve on the target's surface. Current MLS measures targets by obtaining the range and angle measurements. The range measurements by RIEGL scanners are determined by the time-of-flight (TOF) method, i.e. the scanner sends a short laser pulse to the surrounding environment, once it hits a target and returns, the time difference between the emitted pulse and returned pulse is measured to determine the range $R$ :

$$
R=\frac{1}{2} c \Delta t
$$


where $c$ is the speed of light and $\Delta t$ is the TOF of the pulse. The laser range and angle measurements are stored in the scanner local coordinate system which are then integrated with the position and orientation of the laser scanner measured by navigation systems to produce the 3D coordinates of the targets captured by the laser pulses in a geodetic global coordinate system. Laser scanners are characterised by many aspects including those listed in Table 1.

Another important feature in modern laser scanners is the capability of identifying multiple targets from the same laser pulse, measured by multi-target resolution (MTR), which is characterised by the minimum distance between the two targets (Guarnieri et al., 2012). This is especially important when scanning forestry environments, where users are not only interested in the canopy, but also the terrain and vegetation. Without the ability of multitarget detection, objects hidden by the trees would not be picked up by the laser scanner. For better multiple target detection with high accuracy, the process of echo digitisation and waveform processing is essential. Echo digitisation is the process of sampling signals at a high sampling rate and converting them to a digital representation. Once the signals are digitised, a full waveform analysis (FWA) is carried out based on the assumption that the response signals can be modelled with a Gaussian function and all the backscattering targets are also Gaussian. The Gaussian pulses found in the returned signals are used to performing further analysis (Ullrich and Pfennigbauer, 2011). Both RIEGL scanners here have on-line waveform processing features, which allows better target recognition with higher accuracy in real time. This feature is essential for the study areas discussed above as both are areas with high vegetation and forestry coverage.

\section{GEO-REFERENCING AND ERROR PROPAGATION}

\subsection{Error propagation}

Once the data have been captured, the change detection ability comes down to the accuracy of the captured data, i.e. the level of detectable change is only possible when the it is larger than the specified accuracy. Therefore, the accuracy of the captured data is discussed here to assess the potentials of the mobile mapping in monitoring applications. As given above, the accuracy is fundamentally affected by two factors: the navigation system and the laser scanner.

The position and orientation of the laser scanner is measured by the GNSS and IMU integrated navigation system. Hence the accuracy of the LiDAR system is highly influenced by the navigation accuracy, where both the GNSS and IMU measurements contribute to the navigation error. To achieve better navigation accuracy for kinematic GNSS positioning, Differential GNSS (DGNSS) method is used in post processing to determine the absolute position of the scanner system in the given geodetic reference frame, e.g. WGS84. However, DGNSS positioning error can increase to a few metres under bad satellite conditions and may contain data gaps when the number of visible satellites drops below four. IMU generally comprises 3 accelerometers and 3 gyroscopes to measure the local gravity vector, acceleration vector and angular rate vector. Although the IMU measurements will degrade over time, their relative accuracy in a short period of time, usually within $60 \mathrm{~s}$, is very high. Thus, these measurements are integrated with GNSS measurements to help maintain the positioning accuracy when GNSS accuracy degrades. Ideally, the navigation system used here should give a position accuracy better than $5 \mathrm{~cm}$ RMS and heading accuracy of $0.007^{\circ} \mathrm{RMS}$ in post processing.
The error source from the laser scanner comes from the laser ranging measurement and system calibration. For ranging measurements based on TOF, the accuracy is fundamentally determined by the time measurement of the laser pulses. The time interval measured by current scanners can be up to a precision of $67 \mathrm{ps}$, i.e. a range accuracy of $1 \mathrm{~cm}$. With FWA features, the detection accuracy from laser pulses should be in the millimetre level (Ullrich and Pfennigbauer, 2011). Calibration is carried out before surveys to determine the relative orientation between the laser scanner and the IMU. Therefore, the calibration accuracy will affect how accurate the laser point cloud is in the global reference frame. Yet, calibration can generally achieve millimetre accuracy. Hence the largest error source comes from GNSS positioning.

Measurement errors, or uncertainty, is usually defined by the absolute error $\Delta x$. This is commonly represented by the standard deviation, $\sigma$, which is the positive square root of variance, $\sigma^{2}$. The measurements captured by an MLS can be generalised as a linear combination, denoted as discussed by (Jaboyedoff et al., 2012):

$$
F=A x+B y
$$

where $x$ and $y$ are measurements from independent systems, $A$ and $B$ are their coefficients. Let the variance-covariance matrix on $x$ be denoted as $\Sigma^{x}$,

$$
\Sigma^{x}=\left(\begin{array}{cccc}
\sigma_{1}^{2} & \sigma_{12} & \ldots & \sigma_{1 n} \\
\sigma_{12} & \sigma_{2}^{2} & \ldots & \sigma_{2 n} \\
\vdots & \vdots & \vdots & \vdots \\
\sigma_{1 n} & \sigma_{2 n} & \ldots & \sigma_{n}^{2}
\end{array}\right)
$$

whereas $\sigma_{n}^{2}$ is the variance of the $n$th variable, $\sigma_{m n}$ is the covariance between variables $n$ and $m$.

We can assume that errors in the navigation system and the scanner system will be propagated linearly to the point cloud in MLS measurements. The standard deviation of function $f$ is expressed as,

$$
\sigma_{f}=\sqrt{A^{2} \sigma_{x}^{2}+2 A B \sigma_{x y}+B^{2} \sigma_{y}^{2}}
$$

As $x, y$ are uncorrelated, the covariance term $\sigma_{x y}=0$. The standard deviation can be simplified as

$$
\sigma_{f}=A \sigma_{x}+B \sigma_{y}
$$

As the errors coming from the laser scanner are negligible compared to the navigation errors, we can assume that the errors seen in the point cloud should be around the same error level as the positioning system. Hence, the errors in the geo-referencing must be reduced to its minimum to produce the best possible point cloud for change detection. Two ways of improving accuracy are to improve positioning accuracy itself and to improve the relative accuracy in the point cloud.

\subsection{Navigation accuracy}

To achieve the best navigation accuracy, usually a surveying grade GNSS is integrated with at least a tactical grade IMU. The IMU will is able to maintain millimetre accuracy for a few seconds when GNSS signal degrades. The system accuracy will reduce exponentially as the poor signal period grows. One of the most common methods for ensuring accuracy and reliability is to place control points at certain locations within the target area. These are static and easy to find targets which are surveyed to within $1 \mathrm{~cm}$ accuracy. During post processing, the point cloud is registered by finding the control points and adjusting the whole data 


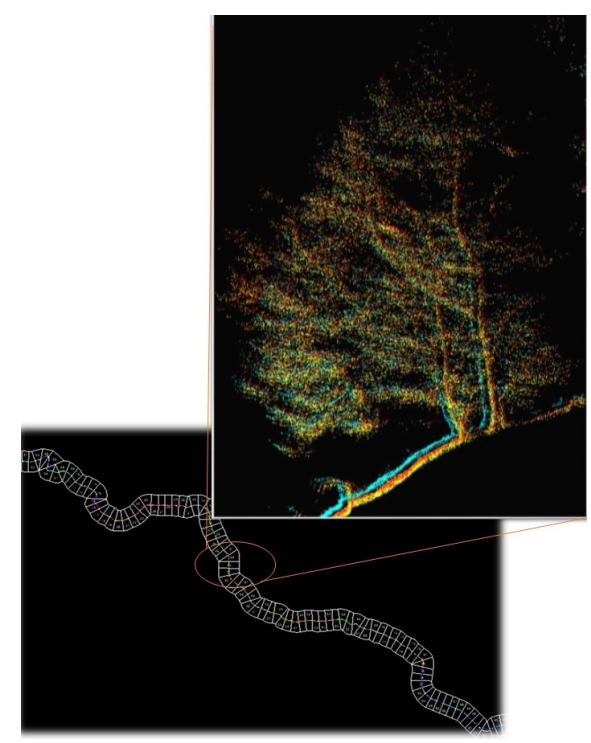

Figure 3: Example of point cloud mismatch

according to these measured coordinates.

The navigation accuracy in the first study area should be ideal as the system was installed in open sky. The GNSS signals should be optimal in such conditions without any disturbance and blockage. Control points were also used in this study as this was a designated study area for repeated scans and the area was a reasonable size to place targets.

The navigation situation in the second study area is more challenging, where the LiDAR system is installed on a vehicle that drives through a country road which is partly covered by foliage. There are two long periods of GNSS data outage as the system lose signals for more than a minute. No control points were used during the data capture. This simulates a real data capture scenario for mobile monitoring applications over large and remote areas, where it is very unlikely to place control points for a project that covers a large area.

For both datasets, the GNSS data is processed in Inertial Explorer to produce navigation results with a standard deviation within 10 $\mathrm{cm}$ in the horizontal plane and $20 \mathrm{~cm}$ in the vertical direction. These results are then integrated with the IMU measurements in AeroOffice, the navigation processing software by IGI. During this step, static periods and the periods where GNSS measurements differs with IMU measurements are marked out to correct navigation accuracy with IMU measurements. This will ensure that the navigation data is processed to its best possible accuracy by using only the optimal GNSS performance and IMU performance during different periods.

The final integrated navigation solution is exported with a data rate of $256 \mathrm{~Hz}$, which is then integrated with the laser measurements to geo-reference the point cloud.

\subsection{Point cloud}

Due to the poor GNSS conditions during data capture in the second study area, the points representing the same location but captured at a different time do not completely overlap. The worst sections of the point cloud have a mismatch of up to $30 \mathrm{~cm}$, as shown in Figure 3. Therefore, before the data can be used for change analysis, the relative accuracy of the point clouds needs to be adjusted using point cloud processing tools, e.g. TerraScan

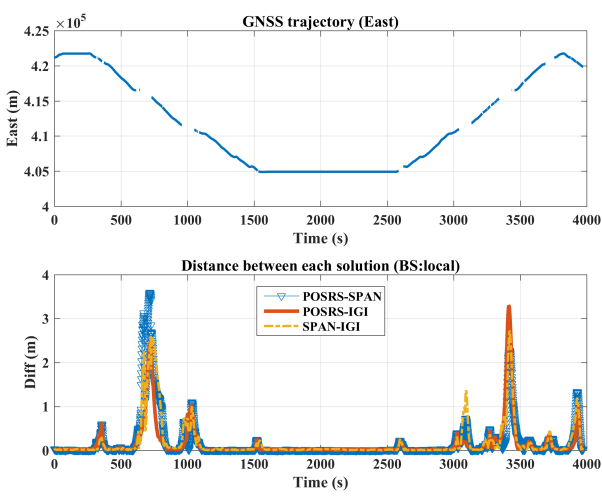

Figure 4: Positioning results from different navigation systems

and TerraMatch. This can be used to adjust the point cloud data within the same project and also adjust the point cloud for the same area but collected in different projects. The method applied here is to register the point clouds from different capture times by manually finding distinct common features in the point clouds (e.g. sign posts or corner of houses) and matching them up. As a result, the point clouds captured at different periods will overlap regardless of its positioning accuracy.

For an automated process to register the point cloud captured in different projects, the fine registration method in CloudCompare is used. This method is based on the Iterative Closest Points (ICP) algorithm, where for each point from a point cloud, the source cloud, the closest point in another point cloud, the reference cloud, is found to minimise the distance between the two clouds. A combination of rotation and translation is estimated to align each source point to the reference and the source points are transformed using the obtained transformation matrix. This process is iterated until the distance between the two point clouds is minimised. This method is a much faster method to register the point cloud, although at the cost of smoothing a regional error over the entire dataset. Fine registration is carried out before change detection between two sections of the point cloud data which cover the same area but captured at different times. This is to ensure that the two datasets used to detect change completely overlap.

However, the absolute accuracy in both methods may partly be lost due to the way the point clouds are registered relatively to each other rather than an absolute position.

\section{RESULTS AND ANALYSIS}

\subsection{Geo-referencing}

To compare the navigation accuracy that can be achieved in difficult environments such as Snake Pass, the StreetMapper system was installed on an experimental van from the Nottingham Geospatial Institute, The University of Nottingham. Two high grade inertial navigation systems (INS) are fixed on the van to provide a position reference, i.e. NovAtel SPAN LCI IMU and Applanix Commercial IMU (POS RS) both integrated with NovAtel GNSS receivers. Positioning accuracy specifications are listed in Table 2.

Based on the specifications, the Applanix POS RS system gives the best positioning performance. Hence the results from all other systems are evaluated by comparing to the results from POS RS, as shown in Figure 4 . It can be clearly seen that during the GNSS signal outage periods, the positioning results from different systems differ by up to $3 \mathrm{~m}$ or more. The standard deviation given 
Table 2: INS accuracy specifications

\begin{tabular}{c|c|c|c|c|c}
\hline & Outage & Horizontal $(\mathrm{m})$ & Vertical $(\mathrm{m})$ & Heading $(\mathrm{deg})$ & Roll/Pitch (deg) \\
\hline \multirow{2}{*}{ SPAN } & $0 \mathrm{~s}$ & 0.02 & 0.05 & 0.018 & 0.007 \\
& $60 \mathrm{~s}$ & 1.67 & 0.48 & 0.021 & 0.009 \\
\hline \multirow{2}{*}{ POS RS } & $0 \mathrm{~s}$ & 0.008 & 0.015 & 0.025 & 0.003 \\
& $60 \mathrm{~s}$ & 0.5 & $/$ & 0.03 & 0.04 \\
\hline
\end{tabular}

Table 3: Positioning results by using different base station data

\begin{tabular}{c|cc|c}
\hline \multirow{2}{*}{ Base station } & \multicolumn{2}{|c|}{ Errors (m) } & \multirow{2}{*}{ Data outage } \\
& Mean & Std & \\
\hline Local & 0.45 & 1.82 & $14.5 \%$ \\
OS & 0.63 & 2.27 & $8.5 \%$ \\
Virtual & 0.64 & 2.26 & $8.6 \%$ \\
\hline
\end{tabular}



Figure 5: Navigation standard deviation compared with point cloud mismatch

by the processing software during these periods are shown in Figure 5. The mismatch seen in the actual point clouds during these periods are also shown in the figure.

For better positioning accuracy, DGNSS was used in post-processing, which requires a base station to be set up near the trial location. However, as mentioned above, setting up physical dedicated base stations is not the best approach in real scenarios. Therefore, positioning results achieved by using base station corrections downloaded from the Ordnance Survey (OS) Net, a network of GNSS base stations covering Great Britain, is compared here. Another advantage of the OS Net data is that, although the actual network base stations can be more then $40 \mathrm{~km}$ apart, users can generate virtual base stations which can be located next to the project location, based on the data from the network. The mean and standard deviation of errors from using a physical local base station, an OS Net station and a virtual station are listed in Table 3.

As shown, using the OS base station data gives similar performance to using the local base station. For a project that covers an area around the size of the Snake Pass project, using virtual base stations does not give significant advantage over using the OS net base stations which are up to $40 \mathrm{~km}$ away from the project location. The obtained geo-referencing solution is best possible solution for near-real time processing without further corrections. Hence it used for geo-referencing in the next steps.

\subsection{Change detection}

As discussed above, the accuracy of the point cloud is highly dependent on the accuracy of the navigation system. However, the system will unavoidably need to scan in difficult environments
Table 4: Change detection level from point clouds (m)

\begin{tabular}{c|cc|cc}
\hline \multirow{2}{*}{ Data section } & \multicolumn{2}{|c|}{ Before corrections } & \multicolumn{2}{c}{ After corrections } \\
& mean & std & mean & std \\
\hline 1 & 0.148 & 0.505 & 0.026 & 0.048 \\
2 & 0.122 & 0.161 & 0.117 & 0.110 \\
3 & 0.126 & 0.187 & 0.087 & 0.085 \\
4 & 0.088 & 0.279 & 0.071 & 0.102 \\
\hline
\end{tabular}

in a long term and long distance monitoring project, where centimetre level accuracy is hard to maintain. Hence, obtaining high relative accuracy between the point clouds is a vital step before change detection analysis.

The point clouds obtained from the above steps are used here to help determine the level of change that can be detected. The data from the first study area is analysed to show the surface displacement velocity, which firstly needs to be classified on the onlinewaveform laser data to distinguish bare earth and vegetated terrain. To limit the impact from detecting changes in vegetation growth rather than the terrain features when comparing data, only the data classified as bare earth are used for analysis. Figure $6 \mathrm{a}$ shows a set of automatically detected surface features used for tracking surface change. The tracked terrain displacement between two datasets are shown in Figure 6b, where there are some large displacement vectors caused by falsely using vegetation features. Although the classification is generally quite accurate, even minor errors could lead to false detection.

The second study area is analysed for change detection of larger displacements as the geo-referencing accuracy does not allow displacement computation on very high level. The change analysis is achieved by calculating the cloud-to-cloud distance. This function computes the approximate distances between two point cloud datasets (a source and a reference cloud) captured at different periods by creating model planes of the two clouds using least squares best fit. For each point in the source cloud, its nearest point in the reference cloud is determined and a local model is fitted on the nearest point and its neighbours. The distance is then computed from the source cloud point to this reference model plane rather than the reference point. This is less dependent on the density of the point cloud and can produce better results on the global scale (CloudCompare, 2016). Figure 7 shows the cloud-to-cloud distance computation results before and after

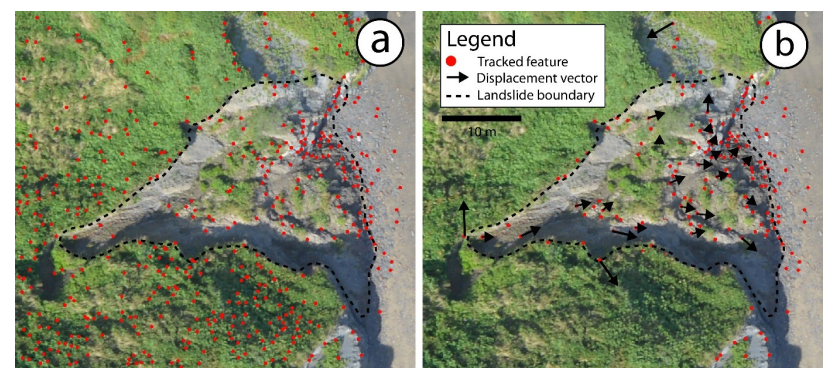

Figure 6: (a) Selected features in the study area for surface tracking (b) Computed terrain displacement 


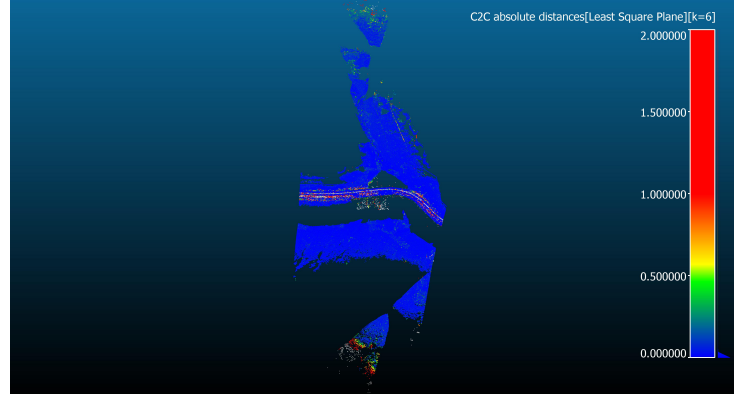

(a) Before corrections

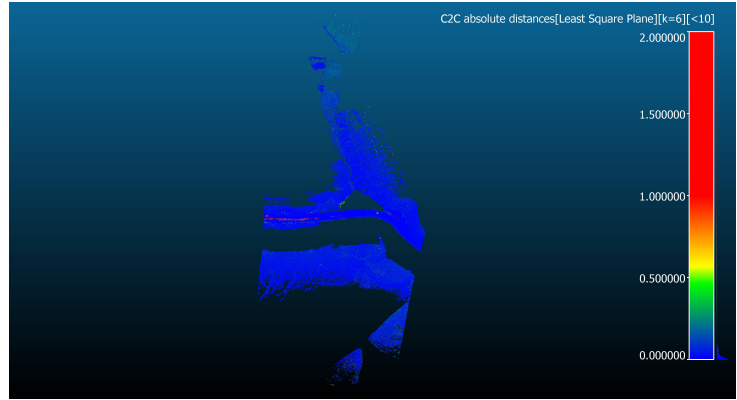

(b) After corrections

Figure 7: Change analysis from cloud-to-cloud distance

applying the point cloud relative corrections described in Section 3.3 .

The mean and standard deviation of the computed change obtained from the four sections of the point cloud data are listed in Table 4. As shown, the distance is reduced after applying corrections, indicating that any mismatch in the data will appear as change in the final computation results. However, the mismatch may come from both actual change and false change. Some examples for false change include vegetation change, temporary targets such as vehicles and pedestrians, geo-reference errors and errors from different data boundaries which will also cause the data to not completely overlap. Change detection validity is confirmed by visually inspecting and identifying changes between the point clouds against the computation results. Figure 8a shows an aerial view of the area which appears as change in the cloud-to-cloud computation. On the right-hand side, the side-view of the point cloud data in the area from two different time periods are shown, which shows a part of a wall that has fallen down between two data capture times. However, the computed change in this area



(a) Cloud-to-cloud distance measurement is around $55 \mathrm{~cm}$, which is the same level as other detected false change.

From the change analysis carried out on the Snake Pass dataset, we can see that after applying relative corrections to the point cloud data, changes above the level of $10 \mathrm{~cm}$ can be detected by applying cloud-to-cloud distance computation. However, the danger in simple distance computation is that false detection is picked up as well as real changes in the environment.

\section{CONCLUSIONS AND FUTURE WORK}

This paper presents preliminary results of remote area change detection and monitoring using mobile mapping systems. By using high resolution 2D laser scanners with online-waveform analysis integrated high grade navigation systems, the mapping system is able to achieve decimetre level accuracy in most areas. However, the geo-referencing accuracy will degrade when GNSS signals are degraded or blocked for a long time, i.e. over a minute. Hence introducing errors in the point cloud. For better change detection analysis, the relative accuracy of the point cloud is improved by correcting the point cloud itself so that the data captured at the same location will overlap regardless of absolute positioning accuracy in the global reference system.

With the ability to detect different terrains from the laser data, better surface displacement can be achieved. The monitored area is estimated with a surface displacement of $\sim 2.5 \mathrm{~m} / \mathrm{yr}$ using automatic detection and computation approaches. However, more sophisticated terrain classification and tracking methods should be tested in the future to eliminate detection errors.

For change analysis in more difficult environments, relative changes that are above $10 \mathrm{~cm}$ level can also be obtained by computing cloud-to-cloud distance. However, different change results should be analysed to form a change detection classification method. This will allow automatic classification of valid and false change detection in the future where false detections will not show up in the final results and no human intervention is required during the processing.

\section{ACKNOWLEDGEMENTS}

The authors would like to thank the Nottingham Geospatial Institute, The University of Nottingham for providing the experimental facilities. This project is co-funded by Innovative UK as part of the Knowledge Transfer Partnership programme.

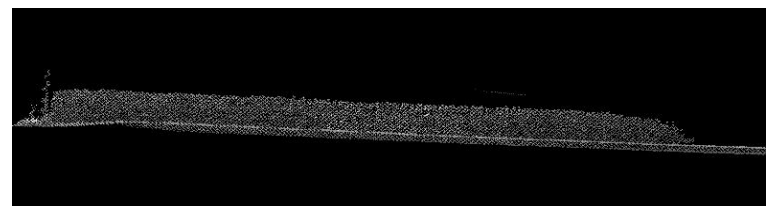

(b) Captured data 1

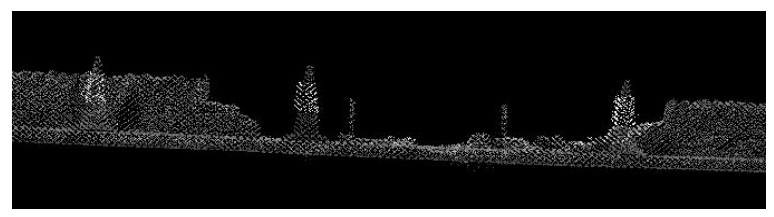

(c) Captured data 2

Figure 8: Change detection example showing road-side infrastructure damage 


\section{REFERENCES}

Bitenc, M., Lindenbergh, R., Khoshelham, K. and van Waarden, A. P., 2011. Evaluation of a LIDAR land-based mobile mapping system for monitoring sandy coasts. Remote Sensing 3(7), pp. 1472-1491.

Boon, D. and Evans, H., 2008. Walkover survey of a landslide on the A57 (Snake Pass) road at Cowms Moor, Derbyshire in January 2008. BGS Land Use and Development Open Report OR/08/006.

CloudCompare, 2016. CloudCompare User Manual Version 2.6.1. http://cloudcompare.org/. (March 2016).

González-Jorge, H., Arias Sanchez, P., Puente, I. and Martínez, J., 2012. Surveying of road slopes using mobile LiDAR. Gerontechnology 11(2), pp. 1-4.

Guarnieri, A., Pirotti, F. and Vettore, A., 2012. Comparison of Discrete Return and Waveform Terrestrial Laser Scanning for Dense Vegetation Filtering. ISPRS - International Archives of the Photogrammetry, Remote Sensing and Spatial Information Sciences XXXIX-B7(September), pp. 511-516.

Jaboyedoff, M., Oppikofer, T., Abellán, A., Derron, M. H., Loye, A., Metzger, R. and Pedrazzini, A., 2012. Use of LIDAR in landslide investigations: A review. Natural Hazards 61(1), pp. 5-28.

Kaartinen, H., Hyyppä, J., Kukko, A., Jaakkola, A. and Hyyppä, H., 2012. Benchmarking the Performance of Mobile Laser Scanning Systems Using a Permanent Test Field. Sensors 12(12), pp. 12814-12835.

Kukko, A., Kaartinen, H., Kaasalainen, S., Anttila, K. and Vaaja, M., 2010. Experiences of mobile mapping in environmental monitoring. Ubiquitous Positioning Indoor Navigation and Location Based Service (UPINLBS 2010), pp. 1-6.

Lindenbergh, R. and Pietrzyk, P., 2015. Change detection and deformation analysis using static and mobile laser scanning. Applied Geomatics 2015(7), pp. 65-74.

Park, H. S., Lee, H. M., Adeli, H. and Lee, I., 2007. A new approach for health monitoring of structures: Terrestrial laser scanning. Computer-Aided Civil and Infrastructure Engineering 22(1), pp. 19-30.

Slatcher, S., Gonzalez, C., Cox, C. and Starka, L., 2015. Quantifying coastal slope instability through integrated time-series airborne laser scanning and photography. MMT 2015 (9th International Symposium on Mobile Mapping Technology).

Ullrich, A. and Pfennigbauer, M., 2011. Echo digitization and waveform analysis in airborne and terrestrial laser scanning. Photogrammetric Week, pp. 217-228.

Vu, T., Matsuoka, M. and Yamazaki, F., 2004. LIDAR-based change detection of buildings in dense urban areas. 2004 IEEE International Geoscience and Remote Sensing Symposium Proceedings, IGARSS '04. 5, pp. 3413-3416.

Xiao, W., Vallet, B. and Paparoditis, N., 2013. Change Detection in 3D Point Clouds Acquired by a Mobile Mapping System. ISPRS Annals of Photogrammetry, Remote Sensing and Spatial Information Sciences, II-5/W2(November), pp. 331-336. 